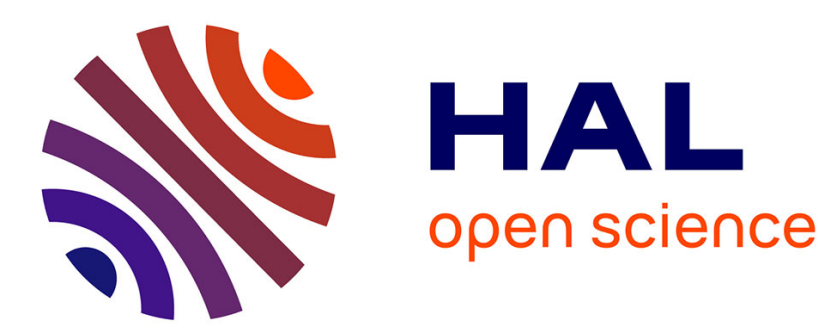

\title{
R2-EMOA: Focused Multiobjective Search Using R2-Indicator-Based Selection
}

Heike Trautmann, Tobias Wagner, Dimo Brockhoff

\section{To cite this version:}

Heike Trautmann, Tobias Wagner, Dimo Brockhoff. R2-EMOA: Focused Multiobjective Search Using R2-Indicator-Based Selection. Learning and Intelligent OptimizatioN Conference (LION 7), Jan 2013, Catania, Italy. pp.70-74, 10.1007/978-3-642-44973-4_8 . hal-00807901

\section{HAL Id: hal-00807901 https://hal.inria.fr/hal-00807901}

Submitted on 25 Mar 2014

HAL is a multi-disciplinary open access archive for the deposit and dissemination of scientific research documents, whether they are published or not. The documents may come from teaching and research institutions in France or abroad, or from public or private research centers.
L'archive ouverte pluridisciplinaire HAL, est destinée au dépôt et à la diffusion de documents scientifiques de niveau recherche, publiés ou non, émanant des établissements d'enseignement et de recherche français ou étrangers, des laboratoires publics ou privés. 


\title{
R2-EMOA: Focused Multiobjective Search Using R2-Indicator-Based Selection*
}

\author{
Heike Trautmann ${ }^{1}$, Tobias Wagner ${ }^{2}$, and Dimo Brockhoff ${ }^{3}$ \\ 1 Statistics Faculty, TU Dortmund University, Germany \\ trautmann@statistik.tu-dortmund.de \\ 2 Institute of Machining Technology, TU Dortmund University, Germany \\ wagner@isf.de \\ 3 INRIA Lille Nord-Europe, Dolphin Team, Villeneuve d'Ascq, France \\ dimo.brockhoff@inria.fr
}

\begin{abstract}
An indicator-based evolutionary multiobjective optimization algorithm (EMOA) is introduced which incorporates the contribution to the unary R2-indicator as the secondary selection criterion. First experiments indicate that the R2-EMOA accurately approximates the Pareto front of the considered continuous multiobjective optimization problems. Furthermore, decision makers' preferences can be included by adjusting the weight vector distributions of the indicator which results in a focused search behavior.
\end{abstract}

Keywords: Multiobjective Optimization, Performance Assessment, EMOA, R2-Indicator, Indicator-Based Selection, Preferences

\section{Introduction}

Throughout this paper, we consider multiobjective optimization problems consisting of $d$ objectives $Y_{j}$ and objective functions $f_{j}: \mathbb{R}^{n} \rightarrow \mathbb{R}$ with $1 \leq j \leq d$. In the context of performance assessment of multiobjective optimizers, the (binary) R-indicator family was introduced by Hansen and Jaszkiewicz [5]. It is based on a set of utility functions. In total, three different variants were proposed which differ in the way the utilities are evaluated and combined - the ratio of one set being better than the other (R1), the mean difference in utilities (R2), or the mean relative difference in utilities (R3). In particular, the second variant R2 is one of the most recommended performance indicators [8] together with the hypervolume (HV, [9]) which directly measures the dominated objective hypervolume bounded by a reference point dominated by all solutions. Recently, we defined an equivalent unary version of this R2 indicator [3]. In case the standard weighted Tchebycheff utility function with ideal point $\mathbf{i}$ is used, it is defined as

$$
\operatorname{R} 2(A, \Lambda, \mathbf{i})=\frac{1}{|\Lambda|} \sum_{\lambda \in \Lambda} \min _{a \in A}\left\{\max _{j \in\{1, \ldots, d\}}\left\{\lambda_{j}\left|\mathbf{i}_{j}-a_{j}\right|\right\}\right\}
$$

\footnotetext{
* This is an author version of the LION'2013 short paper published by Springer Verlag. The final publication is available at www.springerlink.com.
} 
for a solution set $A$ and a given set of weight vectors $\lambda=\left(\lambda_{1}, \ldots, \lambda_{d}\right) \in \Lambda$.

Theoretical and experimental comparisons to the HV for $d=2$ revealed that, contrarily to common assumptions, the R2 indicator even has a stronger bias towards the center of the Pareto front than the HV [3]. Furthermore, it could be proven that for $d=2$ the optimal placement of a point w.r.t. the R2indicator solely depends on its two nearest neighbors and a subset of $\Lambda$. In [6], the influence of the R2-indicator parametrization on the optimal distribution of $\mu$ points on the true Pareto front $(\mathrm{PF})$ regarding $\mathrm{R} 2$ was investigated. It was shown that this distribution heavily depends on the position of the ideal point, as well as on the domain and distribution of the weight vectors. Thus, preferences of the decision maker can be reflected by a specifically parametrized R2-indicator. In [1] a similar approach relying on linear utility functions was used to identify knees of Pareto fronts. In this paper, we will investigate whether the approximated optimal distributions of $\mu$ points regarding $\mathrm{R} 2$ based on different preference articulations [6] can be accurately reproduced by a greedy R2-EMOA.

\section{R2-EMOA}

The proposed R2-EMOA implements a steady state strategy based on the contribution to the unary R2-indicator (see Alg. 1).

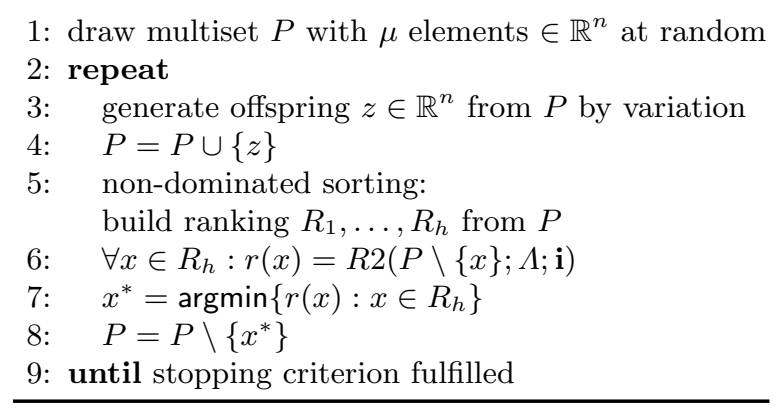

Algorithm 1: Pseudo code of the R2-EMOA.

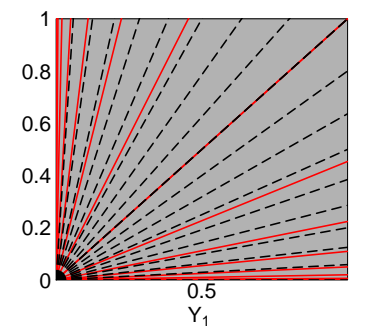

Fig. 1: $|\Lambda|=19$ for $\gamma=$ 1 (black dashed line) and $\gamma=4$ (red solid line).

\section{$3 \quad$ Experiments}

Experiments were conducted to empirically show that the evolutionary procedure (selection pressure, variation) of the R2-EMOA is adequate to accurately approximate the R2-optimal distributions. This cannot be directly assumed, as the greedy strategy of the EMOA which only changes single solutions could be 

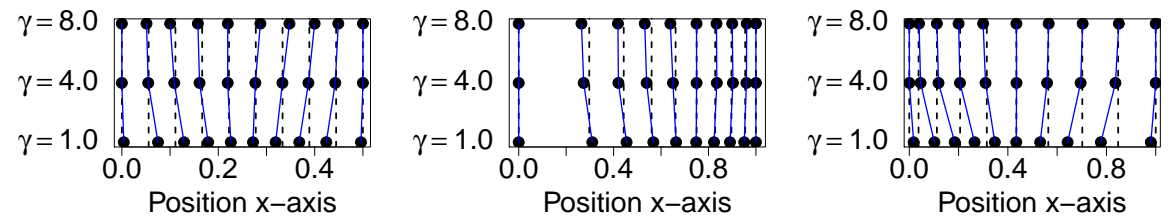

Fig. 2: Results of best R2-EMOA runs for increasing $\gamma$ on DTLZ1 (left), DTLZ2 (middle) and ZDT1 (right). The movement of the $x$-axis positions for $\gamma \in\{1,4,8\}$ is shown. The optimal distributions regarding $\mathrm{HV}$ are reflected by dashed vertical lines.

stuck in local optima of the objective functions or in suboptimal distributions ${ }^{4}$.

For the experiments, three bi-objective test functions with different problem characteristics were selected: ZDT1 (convex PF, $n=30$ ) [7], DTLZ1 (linear PF, $n=6$ ), and DTLZ2 (concave PF, $n=11$ ) [4]. On each function, ten independent runs were conducted using simulated binary crossover (SBX) and polynomial mutation $\left(p_{c}=0.9, p_{m}=1 / n, \eta_{c}=15, \eta_{m}=20\right), 150.000$ function evaluations (FE), ideal point $\mathbf{i}=(0,0)^{\prime}$, and 501 weight vectors. A population size of $\mu=10$ was chosen in order to allow a clear visualization of the results and the comparison to the reference distributions of [6].

The influence of restricted weight vector domains and altered weight vector distributions on the outcome of the R2-EMOA results is considered. Therefore, Alg. 1 of [6] was used to generate weight vector distributions with increasing focus on the extremes of the weight vector domain (see Fig. 1). This is reflected by an increased value of $\gamma$ while $\gamma=1$ corresponds to equally distributed weight vectors in $[0,1]^{2}$. The R2-EMOA is able to accurately approximate the optimal distributions. With increasing $\gamma$, the points tend to drift towards the extremes of the front (Fig. 2) which is perfectly in line with the results of [6]. Individually for each problem, distributions close to the optimal ones regarding HV can be obtained for a specific choice of $\gamma$.

Moreover, the first component of the weight vector domain was restricted to one or two intervals within $[0,1]$. From $[6]$ it is known that in this setting the optimal solutions regarding $\mathrm{R} 2$ lie within the target cone defined by the two outmost weight vectors of the interval(s). This is reflected by the respective R2-EMOA results (Fig. 3).

Fig. 4 relates the final $\mathrm{R} 2$ values of all experiments to the approximated optimal 10-distributions regarding R2 [6]. It can be observed that the variance of the R2-EMOA results is small. Sometimes even slightly better approximations of the optimal distributions are obtained than in [6]. This is rather surprising as these reference solutions were determined based on a global optimization on the front. The evolutionary mechanism and the greedy selection seem to provide efficient heuristics for the considered class of problems.

\footnotetext{
${ }^{4}$ For the HV indicator, it has been, for example, theoretically proven that such a greedy strategy cannot always find a solution set with optimal HV value [10,2].
} 

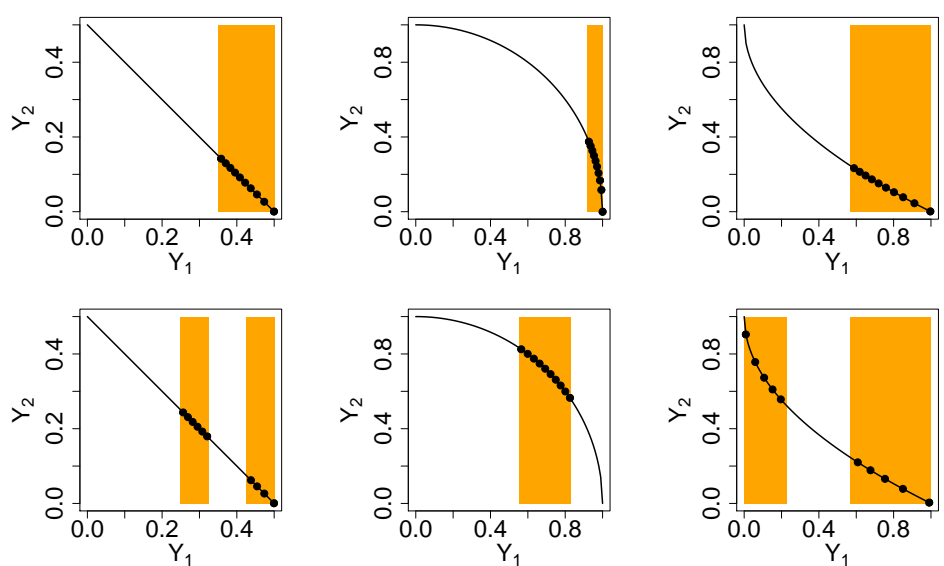

Fig. 3: Results of the best R2-EMOA runs (black dots) with restricted weight vector domains for DTLZ1 (left), DTLZ2 (middle) and ZDT1 (right). The areas within the intersections with the true PF (solid line) are highlighted.
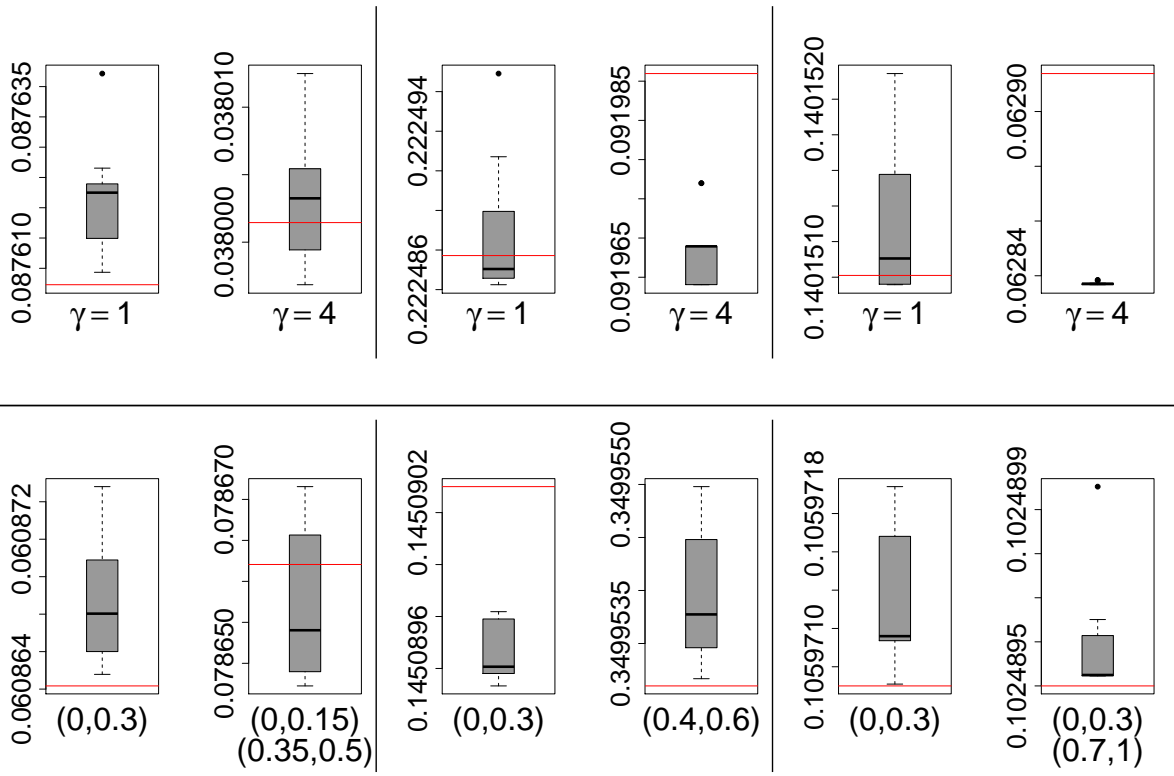

Fig. 4: Boxplots of R2 values at final R2-EMOA generation for DTLZ1 (left), DTLZ2 (middle) and ZDT1 (right) for altered weight distributions with parameter $\gamma$ (top) or restricted weight space (bottom) corresponding to Fig. 3. The R2 value of the approximated optimal 10-distribution of R2 in [6] is visualized by a red horizontal line. 


\section{Conclusions and Outlook}

First experiments show very promising results of the R2-EMOA regarding solution quality and the possibility of incorporating preferences of the decision maker. In future studies, the R2-EMOA will be theoretically and empirically compared to other EMOA optimizing the R2-indicator, such as MOEA/D and MSOPS. Furthermore, theoretical derivations of optimal distributions of $\mu$ solutions regarding $\mathrm{R} 2$ are needed.

\section{Acknowledgements.}

This paper is based on investigations of the project D5 "Synthesis and multiobjective model-based optimization of process chains for manufacturing parts with functionally graded properties" as part of the collaborative research center SFB/TR TRR 30 and the project B4 of the Collaborative Research Center SFB 823, which are kindly supported by the Deutsche Forschungsgemeinschaft (DFG). In addition, the authors acknowledge support by the French national research agency (ANR) within the Modèles Numérique project "NumBBO - Analysis, Improvement and Evaluation of Numerical Blackbox Optimizers" (ANR12-MONU-0009-03).

\section{References}

1. Branke, J., Deb, K., Dierolf, H., Osswald, M.: Finding knees in multi-objective optimization. In: Conference on Parallel Problem Solving from Nature (PPSN VIII). pp. 722-731. LNCS, Springer (2004)

2. Bringmann, K., Friedrich, T.: Convergence of Hypervolume-based Archiving Algorithms I: Effectiveness. In: Genetic and Evolutionary Computation Conference (GECCO 2011). pp. 745-752. ACM (2011)

3. Brockhoff, D., Trautmann, H., Wagner, T.: On the Properties of the $R 2$ Indicator. In: Genetic and Evolutionary Computation Conference (GECCO 2012). pp. 465$472(2012)$

4. Deb, K., Thiele, L., Laumanns, M., Zitzler, E.: Scalable Multi-Objective Optimization Test Problems. In: Congress on Evolutionary Computation (CEC 2002). pp. 825-830. IEEE Press (2002)

5. Hansen, M.P., Jaszkiewicz, A.: Evaluating The Quality of Approximations of the Non-Dominated Set. Tech. rep., Institute of Mathematical Modeling, Technical University of Denmark (1998), iMM Technical Report IMM-REP-1998-7

6. Wagner, T., Trautmann, H., Brockhoff, D.: Preference Articulation by Means of the $R 2$ Indicator. In: Evolutionary Multiobjective Optimization Conference (EMO 2013). p. accepted (2013)

7. Zitzler, E., Deb, K., Thiele, L.: Comparison of Multiobjective Evolutionary Algorithms: Empirical Results. Evolutionary Computation 8(2), 173-195 (2000)

8. Zitzler, E., Knowles, J., Thiele, L.: Quality assessment of Pareto set approximations. In: Branke, J., Deb, K., Miettinen, K., Slowinski, R. (eds.) Multiobjective Optimization, Lecture Notes in Computer Science, vol. 5252, pp. 373-404. Springer, Berlin / Heidelberg (2008) 
9. Zitzler, E., Thiele, L.: Multiobjective Optimization Using Evolutionary Algorithms - A Comparative Case Study. In: Conference on Parallel Problem Solving from Nature (PPSN V). pp. 292-301. LNCS, Springer (1998)

10. Zitzler, E., Thiele, L., Bader, J.: On Set-Based Multiobjective Optimization. IEEE Transactions on Evolutionary Computation 14(1), 58-79 (2010) 